\title{
Erratum: Measurement of the reactor antineutrino flux and spectrum at Daya Bay [Phys. Rev. Lett. 116, 061801 (2016)]
}

F. P. An et al.

\author{
(Daya Bay Collaboration)
}

(Received 27 January 2017; published 1 March 2017)

DOI: 10.1103/PhysRevLett.118.099902

Because of an error in the interpretation of measurement results, the uncertainty on the number of target protons in Table I and in the text was incorrectly reported as $0.47 \%$. The correct uncertainty is $0.92 \%$. As a result, the combined uncertainty is $2.2 \%$, not $2.1 \%$. The uncertainty in the measured IBD yield and flux increases by approximately $0.1 \%$ due to the change in the uncertainty in the number of target protons. The covariance matrix of the extracted reactor antineutrino spectrum in the Supplemental Material has been appropriately corrected. An updated version of the results in this Letter appears in Ref. [1].

[1] F. P. An et al. (Daya Bay Collaboration), Chin. Phys. C 41, 013002 (2017). 